\title{
Quantum search protocol for an atomic array
}

\author{
Marlan O. Scully ${ }^{1,2}$ and M. Suhail Zubairy ${ }^{1,3}$ \\ ${ }^{1}$ Department of Physics, Texas A\&M University, College Station, Texas 77843-4242 \\ ${ }^{2}$ Max-Planck-Institut für Quantenoptik, 85748 Garching, Germany \\ ${ }^{3}$ Department of Electronics, Quaid-i-Azam University, Islamabad, Pakistan
}

(Received 6 October 1999; published 5 July 2001)

\begin{abstract}
Quantum computers can, in principle, exceed the speed of ordinary computers by taking advantage of quantum coherence and entanglement. It is possible to find a "needle in a haystack" of $N-1$ straws in only $\sqrt{N}$ searches by utilizing coherent superposition states. In the present paper we show how modern quantum optics may provide a simple and practicable quantum search procedure, which may also yield insights into quantum search algorithms in general.

DOI: 10.1103/PhysRevA.64.022304

PACS number(s): 03.67.Lx, 42.50.Gy, 42.50.Ar
\end{abstract}

\section{INTRODUCTION}

The potential advantage of quantum computation is illustrated by the problem of searching a quantum database [1-3]. Suppose we are seeking one object out of $N$, e.g., looking for a needle among $N$ straws. Classically we would expect to pick up $N / 2$ straws, on average, before we find the needle. However, as Grover first showed, quantum mechanics allows us to use the superposition principle to find one object out of $N$ in approximately $\sqrt{N}$ trials.

We note that in Grover's original paper entangled states were used. However, entanglement is not a prerequisite for obtaining quantum speedup in database search algorithms. The utility of quantum search procedures is already apparent when we invoke the quantum superposition principle, and it does not require entanglement. In fact, the present work does not involve entangled states, but uses only the concept of coherent superposition states. This distinction was clearly made by Lloyd [4], and was demonstrated in recent experiments [5].

In particular, here we show how modern quantum optics can provide a simple and practicable quantum search procedure. We follow recent quantum optical studies in which it was shown that quantum coherence can be used to produce new effects and devices such as lasing without inversion [6], light pulses moving at a few meters/sec [7], and other linear and nonlinear effects [8]. The basis for these new effects is quantum coherence and interference [9-11]. We now show that these concepts can be used to yield simple implementations of quantum and/or classical search algorithms.

The basic idea and experimental arrangement of the present paper is illustrated in Figs. 1 and 2. In Fig. 1(a) we see a two-dimensional lattice array of atoms, one atom at each $(i, j)$ site. Each atom (lattice site) is coupled via an optical fiber to a particular detector, so that spontaneously emitted radiation from an atom at site $(i, j)$. causes a count in detector $D_{i j}$ [see Fig. 1(b)].

For the present purpose we may consider the atoms to have a simple hydrogen level structure (e.g. $\mathrm{H}, \mathrm{Li}, \mathrm{Na}, \ldots$ ). We begin with $N$ atoms on a lattice, which we optically pump into some nuclear hyperfine level which we denote by $|s\rangle$ ("s" for straw) as in Fig. 2(a). In the interest of simplic- ity, we consider an example of hydrogen atoms, so that $|s\rangle$ $=\left|1 S_{1 / 2}, F=1, m_{F}=-1\right\rangle$, [12]. Next, the "oracle" prepares a particular atom by moving it from $|s\rangle$ to a different hyperfine level $|n\rangle$ (" $n$ " for needle). She accomplishes this by applying two coherent pulses to some specific atom, but we do not know which one. The Hamiltonian for this process is

$$
\begin{aligned}
H(t)= & \hbar \Omega_{1}(x, t)[|a\rangle\langle n|+| n\rangle\langle a|] \\
& +\hbar \Omega_{2}(z, t)[|a\rangle\langle s|+| s\rangle\langle a|],
\end{aligned}
$$

where the notation is explained in Fig. 2. By applying the pulses in the so-called "counterintuitive" sequence $\left(\Omega_{1}\right.$ first followed by $\Omega_{2}$ ) [13], the atom evolves from an initial dark state $|s\rangle$ at $t=0$,

$$
\left|\Psi_{\text {Dark }}(0)\right\rangle=\left.\frac{\Omega_{1}(0)|s\rangle-\Omega_{2}(0)|n\rangle}{\sqrt{\left|\Omega_{1}(0)\right|^{2}+\left|\Omega_{2}(0)\right|^{2}}}\right|_{\Omega_{1}(0) \gg \Omega_{2}(0)} \rightarrow|s\rangle,
$$

which is "dark" in the sense that $H(0)\left|\Psi_{\text {Dark }}(0)\right\rangle=0$; to the final dark state $|n\rangle$ at time $\tau$,

$$
\left|\Psi_{\text {Dark }}(\tau)\right\rangle=\left.\frac{\Omega_{1}(\tau)|s\rangle-\Omega_{2}(\tau)|n\rangle}{\sqrt{\left|\Omega_{1}(\tau)\right|^{2}+\left|\Omega_{2}(\tau)\right|^{2}}}\right|_{\Omega_{2}(\tau) \gg \Omega_{1}(\tau)} \rightarrow-|n\rangle,
$$

for which $H(\tau)\left|\Psi_{\text {Dark }}(0)\right\rangle=0$. The transition from $|s\rangle$ to $|n\rangle$ proceeds, of course, by adiabatically turning the fields $\Omega_{1}(t)$ and $\Omega_{2}(t)$ on and off [13]. We have now set the stage, and proceed to analyze classical and quantum searches for the atom in state $|n\rangle$ among the $N-1$ atoms in state $|s\rangle$.

In order to clearly state the rules of the game, we note that the pulse energy is not a "free" parameter. Following Lloyd [4], we may put a price tag on each search probe shot, call it $\mathcal{C}$. We wish to minimize the cost of needle discoveries. For the usual (classical) search procedure the average cost would be $(N / 2) \mathcal{C}$; but for the present (quantum) search procedure the cost is reduced to $\sqrt{N} \mathcal{C}$. We return to this point in later discussion [following Eq. (7)], where such considerations are tied to experimental limitations and error correction.

We can carry out a classical search by focusing the excitation radiation on each atom in turn. As in Fig. 2(b), we 


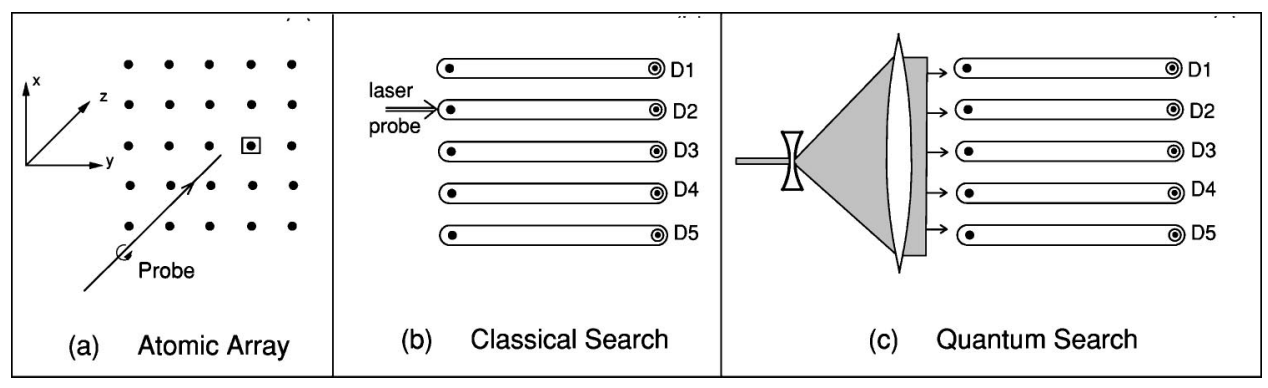

FIG. 1. (a) Atomic array with one specially prepared "needle" atom (in square box) located at an unknown site among $N-1$ other "straw" atoms. A linearly $(z)$ polarized interrogation pulse is incident along the $x$ axis, and a circularly polarized field propagates in the $z$ direction. (b) Atoms in an array are coupled to specific detectors via fiber optical links. In the envisioned experimental situation, fiber is transparent to a radiation exciting atom, as in Fig. 2(b), indicating a two-quantum arrangement. An important point is that fiber contains radiation emitted spontaneously when the atom decays to a detector state manifold as in Fig. 2(b) and is transported to a detector with high efficiency. A classical search is performed when an atom array is probed in a sequential scanning mode such that the laser beam is focused onto each atom in turn. No signal will be observed from straw atoms, since they are not excited by z-polarized radiation; only an atom in the $|n\rangle$ state will be excited, and when this occurs a count in the corresponding detector will be observed. (c) Expanded wave front in which an interrogation pulse is simultaneously incident on the entire atomic array. The electric field will be correspondingly decreased, and a number of excitation pulses is required in order to promote the atom from an $|n\rangle$ state to an excited state.

envision a LCP probe pulse at frequency $\nu_{p}$ and LCP idler radiation at $\nu_{c}$ such that $\nu_{p}+\nu_{c}=\omega_{e, n}[14]$. The pulse duration [15] is adjusted, so that when the probe irradiates an atom in state $|n\rangle$, the atom is excited to state $|e\rangle$ with unit probability. For the moment, let us assume an atom in state $|s\rangle=|0\rangle$ will not be affected by the excitation radiation due to selection rules [16]. The $|e\rangle$ state then decays to $|d\rangle$, and the emitted radiation is registered in the appropriate detector. Thus, when we finally obtain a count in some detector, we know it has been triggered by our $|n\rangle$ atom. Such a classical search procedure will take, on an average, $N / 2$ counts.

However, as we show in the following, we may locate the needle atom in fewer trials by simply optically expanding our probe pulse to cover the entire atomic array as in Fig. 1(c). Such an interaction can produce a coherent superposition in which the needle atom is weakly excited to the $|e\rangle$ state by any one search event. But each search pulse coher- ently adds a small amount to $|e\rangle$. As we shall see, after search events of order $\sqrt{N}$, the needle atom will be found in state $|e\rangle$ with unit probability.

Proceeding with the analysis, the interaction Hamiltonian for the excitation process is

$$
V^{\prime}(t)=\hbar G(t)[|e\rangle\langle n|+\text { adjoint }]
$$

where the two-photon coupling constant is

$$
G=\sum_{i}\left(\wp_{e i} E_{p} / \hbar\right)\left(\wp_{i n} E_{c} / \hbar\right)\left[\left(\nu_{c}-\omega_{i}\right)^{-1}+\left(\nu_{p}-\omega_{i}\right)^{-1}\right]
$$

$\wp_{e i}$ and $\wp_{i n}$ are matrix elements of the dipole moments of the relevant transitions. The coherent idler field $E_{c}$ covers the entire array, and the field strength of the probe laser pulse of

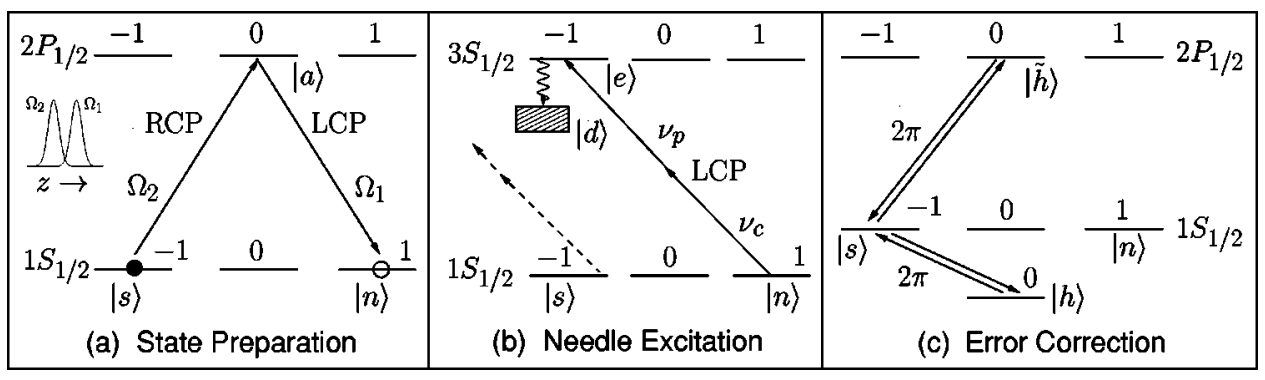

FIG. 2. (a) All $N$ atoms are initially pumped into $|s\rangle m_{F}=0$, and do not respond to the $z$-polarized incident probe field. One atom is now transferred from an $|s\rangle$ state to an $|n\rangle$ state via a counterintuitive pulse sequence, as discussed in the text. (b) A needle atom in state $|n\rangle$ is excited from $1 S_{1 / 2}$ to $3 S_{1 / 2}$ hydrogenlike states via nonlinear two-quantum excitation. The laser field $E_{c}$ is on continuously, whereas the pulsed field at frequency $\nu_{p}$ constitutes the search field. An event is registered when an atom is excited from $|n\rangle$ to $|e\rangle$, and subsequently decays to the $2 P$ manifold of the so-called detector states indicated by $|d\rangle$. A transition from $|1,0\rangle$ to $\left|1^{\prime}, 0^{\prime}\right\rangle$ is forbidden, and this protects straw atoms from excitation, i.e., a dashed line is not allowed. (c) One way of eliminating error counts. Even though $z$ radiation cannot induce an $m_{F}=0$ to $m_{F}=0$ transition by selection rules, spurious environmental effects such as stray electric fields, etc. can perturb the excited state, and transitions could be induced accidentally. One way of protecting straw atoms from such accidental error counts is to sequentially apply $2 \pi$ pulses to an $s$ state (via right circularly polarized radiation) inducing $s \rightarrow h \rightarrow s$ transitions. The atom will now experience a sign change, and an alternate application of excitation pulses followed by $2 \pi$ pulses will lead to a cancellation of accidental counts, as discussed in the text. 
Fig. 1(b) is given by $E_{p}=\sqrt{P / \epsilon_{0} a c}$, where $P$ is the laser power and $a$ is the focused beam area.

The field strength of the expanded beam of Fig. 1(c) is $\mathcal{E}_{p}=\sqrt{P / \epsilon_{0} A c}=E_{p} \sqrt{a / A}$ where $A$ is the expanded beam area. Introducing the atomic density $\rho$ such that $\rho A=N$, we may write $\mathcal{E}_{p}=E_{p} \sqrt{a \rho} / \sqrt{N}=E_{p} / \sqrt{N}$ since $a \rho=1$ for the original focused beam. Substituting $\mathcal{E}_{p}$ for $E_{p}$ in Eq. (3) yields the effective coupling constant $G / \sqrt{N}$ for the expanded beam of Fig. 1(c). The interaction Hamiltonian for the $N$ atom-probe field interaction is then smaller than the focused field expression [Eq. (2)] by $1 / \sqrt{N}$,

$$
V(t)=\frac{\hbar G(t)}{\sqrt{N}}[|e\rangle\langle n|+\operatorname{adj} .] \equiv \frac{\hbar G}{\sqrt{N}} \sigma_{x},
$$

where $\sigma_{x}=|e\rangle\langle n|+| n\rangle\langle e|$. In Eq. (4), the $\sqrt{N}$ factor emphasizes the fact that, expanding the pulse (in order to irradiate the entire array) reduces the electric field "felt" by a single atom.

Thus the atomic state at time $t$ is given by $|\Psi(t)\rangle$ $=U(t)|\Psi(0)\rangle$, where, for our weak expanded pulse, we use Eq. (4), in the large- $N$ limit, to obtain

$$
U(t) \cong 1-\frac{i \phi(t)}{\sqrt{N}} \sigma_{x}
$$

where $\phi\left(t_{1}\right)=\int_{0}^{t_{1}} d t^{\prime} G\left(t^{\prime}\right)$. As discussed earlier, we wish to tailor the focused beam so that it excites the needle atom. For convenience, we choose $\int_{0}^{t_{1}} d t^{\prime} G\left(t^{\prime}\right)=i$, so that $\phi\left(t_{1}\right)=1$.

Applying the pulse $m$ times we have, to a good approximation for large $N$,

$$
U\left(t_{m}\right)=\left(1+\frac{\sigma_{x}}{\sqrt{N}}\right)^{m} \cong 1+\frac{m}{\sqrt{N}} \sigma_{x},
$$

and the probability amplitude for exiting to $|e\rangle$ is

$$
\left\langle e \mid \Psi\left(t_{m}\right)\right\rangle \cong\left\langle e\left|\left(1+\frac{m}{\sqrt{N}} \sigma_{x}\right)\right| n\right\rangle=\frac{m}{\sqrt{N}},
$$

so that when $m \sim \sqrt{N}$ the probability amplitude for exciting the needle atom to state $|e\rangle$ builds up to unity, with all other (straw) atoms remaining in the ground state. State $|e\rangle$ then decays to $|d\rangle$, the emitted light is imaged onto the detector which registers a click, and we have found the needle.

So far we have carried out a perturbative analysis for a weak expanded pulse. In order to prove the result rigorously, we first note that the needle atom state for a "classical" search is

$$
|\psi(t)\rangle=\cos [\phi(t)]|n\rangle-i \sin [\phi(t)]|e\rangle .
$$

We chose $\phi(t)=\pi / 2$ for excitation. Now, if we expand the probe pulse wave-front top cover all $N$ atoms at once, $\phi(t)$ $\rightarrow \phi(t) / \sqrt{N}$, and any given pulse only excites the needle atom a little bit. For $\phi(t)=\pi / 2$, we now have the small Rabi tip angle $\pi / 2 \sqrt{N}$ after the pulse, and we obtain the "buildup" probability in the excited state. The needle atom therefore develops as follows

$$
\text { Start }|\psi(0)\rangle=|n\rangle,
$$

First pulse $|\psi(1)\rangle=\cos (\pi / 2 \sqrt{ } N)|n\rangle-i \sin (\pi / 2 \sqrt{N})|e\rangle$,

$$
\begin{aligned}
\text { Second pulse }|\psi(2)\rangle= & \cos (2 \pi / 2 \sqrt{N})|n\rangle \\
& -i \sin (2 \pi / 2 \sqrt{N})|e\rangle
\end{aligned}
$$

mth pulse $|\psi(m)\rangle=\cos (m \pi / 2 \sqrt{N})|n\rangle-i \sin (m \pi / 2 \sqrt{N})|e\rangle$.

Hence, for $m=\sqrt{N}$, the atom is excited to state $|e\rangle$, and when it decays to $|d\rangle$, we detect the emitted photon and we have found the atom.

One might think that it would be desirable to find the needle in one shot by just turning up the probe laser power. There are several reasons why this is not the optimal protocol and/or is not possible: (1) We have shown, following Lloyd, that the present scheme takes the discovery cost from the classical value of $N / 2 \mathcal{C}$ to the quantum cost of $\sqrt{N} \mathcal{C}$. Increasing the laser power enough to find the needle atom in one shot would require increasing the probe field by $\sqrt{N}$, i.e., increasing the laser power by $N$ and thereby raising the cost to the order of $N \mathcal{C}$, as in the classical case. (2) The one-shot laser power will, for large enough $N$, not be available (at any cost). (3) One-shot discovery precludes our simple error correction procedure, to which we now turn.

From Eq. (7) we see that we have, in principle, found the $|n\rangle$ atom by applying $\sqrt{N}$ pulses. But, in practice, there will always be error counts due to "accidental" excitation of $|s\rangle$ atoms. That is, there will in general be some probability of exciting the $j$ th straw atom to an excited state $\left|f_{j}\right\rangle$, which can decay to $|d\rangle$ thus giving error counts; the $U$ matrix for this process is

$$
U^{j}(\tau)=1+\frac{\delta \phi}{\sqrt{N}}\left[\left|f_{j}\right\rangle\left\langle s_{j}|+| s_{j}\right\rangle\left\langle f_{j}\right|\right]
$$

where $\delta \phi$ is the accidental Rabi angle which is hopefully much smaller than $\phi(t)$. Nevertheless, if $N$ is large enough, these accidental excitations can be compounded into an unacceptably large error count.

Happily, it is possible to reduce (even eliminate) the error counts via a type of "protection" of the $s$ level. As depicted in Fig. 2(c), we envision applying pulses at frequency $\nu_{h s}$ to the atomic array, which would cycle atoms in the $|s\rangle$ states to $|h\rangle$ and back to $|s\rangle$ resulting in a net sign change of the ground state [17].

Selection rules prohibit the excitation of the $|n\rangle$ state by the $2 \pi$ pulses. The $U$ matrix for the $s \rightarrow h \rightarrow s$ evolution of the $j$ th straw atom is given by 


$$
\begin{aligned}
\mathcal{U}^{j}(\Delta t)= & \cos \frac{\Omega \Delta t}{2}\left[\left|s_{j}\right\rangle\left\langle s_{j}|+| h_{j}\right\rangle\left\langle h_{j}\right|\right] \\
& -i \sin \frac{\Omega \Delta t}{2}\left[\left|h_{j}\right\rangle\left\langle s_{j}|+| s_{j}\right\rangle\left\langle h_{j}\right|\right],
\end{aligned}
$$

where $\Omega$ is the coherent drive Rabi frequency.

When $\Omega \Delta t=2 \pi$, we have $\mathcal{U}_{j}(\Delta t)=-\left[\left|s_{j}\right\rangle\left\langle s_{j}\right|\right.$ $\left.+\left|h_{j}\right\rangle\left\langle h_{j}\right|\right]$. We note that atoms in the $\left|s_{j}\right\rangle$ state are not coupled to $\left|f_{j}\right\rangle$ by the $2 \pi$ pulse, and so the complete $\mathcal{U}_{2 \pi}$ matrix for the $j$ th straw atom in this three state space is

$$
\mathcal{U}_{2 \pi}^{j}(\Delta t)=\left|f_{j}\right\rangle\left\langle f_{j}|-| s_{j}\right\rangle\left\langle s_{j}|-| h_{j}\right\rangle\left\langle h_{j}\right| .
$$

Applying the excitation and $j$ th straw atom, we have

$$
U^{j}=\mathcal{U}_{2 \pi}^{j} U\left(t_{m}\right) \mathcal{U}_{2 \pi}^{j} \cdots \mathcal{U}_{2 \pi}^{j} U\left(t_{3}\right) \mathcal{U}_{2 \pi}^{j} U\left(t_{2}\right) \mathcal{U}_{2 \pi}^{j} U\left(t_{1}\right)
$$

now, from Eqs. (8) and (10), we find $\mathcal{U}_{2 \pi}^{j} U\left(t_{l}\right) \mathcal{U}_{2 \pi}^{j}=1$ $-\delta \hat{\phi}^{j}$, where the infinitesimal flip operator $\delta \hat{\phi}^{j} \equiv(\delta \phi /$ $\sqrt{N})\left[\left|f_{j}\right\rangle\left\langle s_{j}|+| s_{j}\right\rangle\left\langle f_{j}\right|\right]$, and Eq. (11) becomes

$$
U^{j}=\left(1-\delta \hat{\phi}^{j}\right) \cdots\left(1+\delta \hat{\phi}^{j}\right)\left(1-\delta \hat{\phi}^{j}\right)\left(1+\delta \hat{\phi}^{j}\right),
$$

Finally, we note that

$$
\left(1-\delta \hat{\phi}^{j}\right)\left(1+\delta \hat{\phi}^{j}\right)=1-\frac{(\delta \phi)^{2}}{N},
$$

i.e., the $2 \pi$ pulsing has essentially eliminated the probability of unwanted excitation of the straw atoms. Hence a count in any given detector can, for all practical purposes, be taken as a "real" (error free) count [18].

To summarize, we have devised a quantum search scheme which reduces the number of search events to $\sqrt{N}$. The key is the use of coherent superposition states such that the probability amplitude for exciting the needle atom is increased by $1 / \sqrt{N}$ in every search event. That is, if $\left|\Psi_{\text {needle }}(m)\right\rangle$ is the state of the needle atom, after $m$ search events, we have

$$
\left|\Psi_{\text {needle }}(m)\right\rangle=\alpha_{m}|e\rangle+\beta_{m}|n\rangle,
$$

where $\alpha_{m}=m / \sqrt{N}$ as in Eq. (7). An essential feature of our search protocol is that we can protect against unwanted straw atom excitation via $2 \pi$ pulse error correction.

The authors acknowledge useful discussions with G. Agarwal, R. Blakely, G. Chen, J. Dowling, B.-G. Englert, E. Farhi, S. Fulling, L. Hau, K. Kapale, A. Klappenecker, H. Lee, S. Lloyd, M. Lukin, A. Matsko, G. Rempe, C. Stroud, B. Stoicheff, I. Walmsley, and S. Yelin; they thank the Office of Naval Research, the National Science Foundation, and the Robert A. Welch Foundation for support.
[1] L. K. Grover, Phys. Rev. Lett. 79, 325 (1997). We note that the present work is solely directed to a search for a specific physical system among many others. It is not about function evaluations or searching a binary database.

[2] See, especially, the elegant treatment of E. Farhi and S. Gutmann, Phys. Rev. A 57, 2403 (1998).

[3] For an excellent discussion of the rapidly developing field of quantum computing, see G. J. Milburn, The Feynman Processor: Quantum Entanglement and the Computing Revolution (Perseus, Reading, MA, 1998). For a discussion of the Grover algorithm, see A. Pittenger, An Introduction to Quantum Computing Algorithms (Birkhäuser Press, Boston, 1999).

[4] S. Lloyd, Phys. Rev. A 61, 010301 (1999).

[5] J. Ahn, T. C. Weinacht, and P. H. Bucksbaum, Science 287, 463 (2000).

[6] See, for example, Laser Phys. 9 (1999), the review issue on LWI, edited by O. Kocharovskaya et al.

[7] S. E. Harris, J. E. Field, and A. Kasapi, Phys. Rev. A 46, R29 (1992); L. V. Hau, S. E. Harris, Z. Dutton, and C. H. Behroozi, Nature (London) 397, 594 (1999); M. M. Kash, V. A. Sautenkov, A. S. Zibrov, L. Hollberg, G. R. Welch, M. D. Lukin, Y. Rostovtsev, E. S. Fry, and M. O. Scully, Phys. Rev. Lett. 82, 5229 (1999); D. Budker, D. Kimball, S. Rochester, and V. Yashchuk, ibid. 83, 1767 (1999).

[8] K. Hakuta, L. Marmet, and B. P. Stoicheff, Phys. Rev. Lett. 66, 596 (1991); M. O. Scully, ibid. 67, 1855 (1991); P. Hemmer, D. P. Katz, J. Donoghue, M. Cronin-Golomb, M. Shahriar, and P. Kumar, Opt. Lett. 20, 982 (1995); M. Jain, M. Xia, G. Y. Yin, A. J. Merriam, and S. E. Harris, Phys. Rev. Lett. 77, 4326
(1996); M. D. Lukin, P. Hemmer, M. Löffler, and M. O. Scully, ibid. 81, 2675 (1998); S. E. Harris and L. V. Hau, ibid. 82, 4611 (1999).

[9] S. E. Harris, Phys. Today 50 (7), 36 (1997).

[10] E. Arimondo, in Progress in Optics edited by E. Wolf (Elsevier, Amsterdam, 1996), Vol. XXXV, p. 257.

[11] M. O. Scully and M. S. Zubairy, Quantum Optics (Cambridge University Press, London, 1997).

[12] In some envisioned experiments, we prefer $\mathrm{Na}$ with a nuclear spin $I=3 / 2$ and therefore an $S_{1 / 2}$ ground state hyperfine state, $F=2$ and 1 , for which $|s\rangle=\left|S_{1 / 2}, F=2, m_{F}=-2\right\rangle$ and $|n\rangle$ $=\left|S_{1 / 2}, F=2, m_{F}=+2\right\rangle$.

[13] J. Oreg, F. T. Hioe, and J. H. Eberly, Phys. Rev. A 29, 690 (1984); J. R. Kuklinski, U. Gaubatz, F. T. Hioe, and K. Bergmann, ibid. 40, 6741 (1989); R. Grobe, F. T. Hioe, and J. H. Eberly, Phys. Rev. Lett. 73, 3183 (1994); A. Kasapi, M. Jain, G. Y. Yin, and S. E. Harris, ibid. 74, 2447 (1995).

[14] As in the papers by W. E. Lamb and co-workers, $\omega$ is an atomic angular frequency and $\nu$ is a radiation angular frequency. We choose a two-photon excitation mechanism so as to arrange for the spontaneously emitted signal photons of Fig. 2(b) to be easily distinguished from the excitation radiation.

[15] We tailor the pulse duration to be small compared to the atomic decay time $|e\rangle \rightarrow|d\rangle$ of Fig. 2(b).

[16] For example, as in Fig. 2(b), the needle atom excitation path is $\left|S, F=1, m_{F}=1\right\rangle \rightarrow\left|P, F=1, m_{F}=0\right\rangle \rightarrow\left|S, F=1, m_{F}=-1\right\rangle$. The straw atoms cannot follow such a path because of selection rules.

[17] This sign change due to a $2 \pi$ rotation of a spinor is, of course, 
well known; i.e., to return to the initial state takes a $4 \pi$ rotation. We could use $21-\mathrm{cm}$ microwave radiation to couple $|S\rangle$ $=\left|S_{1 / 2}, F=1, m_{F}=-1\right\rangle$ to $|h\rangle=\left|S_{1 / 2}, F=0, m_{f}=0\right\rangle$ to affect the $2 \pi$ flips of our straw atoms. Alternately we could $2 \pi$ cycle the straw atoms to higher-lying $n|P\rangle$ states. There are, of course, pros and cons for any arrangement. The important point is that $2 \pi$ error correction is physically possible.
[18] Thus it is clear that the $2 \pi$ error correction is only possible due to the weak probe- $2 \pi$ pulse sequence. If we try to illuminate the entire atomic array with a stronger probe pulse $(N$ times the power in the preset weak probe) in an attempt to locate the needle atom in one shot, there would then be error counts from straw atoms since we no longer have an error correction. 